\title{
Genetic characterization of commensal Escherichia coli isolated from laboratory rodents
}

\author{
Shih Keng Loong ${ }^{1}$, Nur Hidayana Mahfodz ${ }^{1}$, Nurul Asma Anati Che Mat Seri ${ }^{1}$, Haryanti Azura Mohamad Wali², \\ Syahar Amir Abd Gani², Pooi-Fong Wong ${ }^{2,3}$ and Sazaly AbuBakar ${ }^{1,4^{*}}$
}

\begin{abstract}
Background: Escherichia coli, a commensal in the intestines of vertebrates, is capable of colonizing many different hosts and the environment. Commensal E. coli strains are believed to be the precursor of pathogenic strains by means of acquisition of antimicrobial resistant and virulence genes. Laboratory rodents are inherently susceptible to numerous known infectious agents, which could transfer virulence determinants to commensal E. coli. Hence, in this study, the genetic structure of commensal E. coli found in laboratory rodents and their antimicrobial resistance profiles were investigated.
\end{abstract}

Results: E. coli strains belonging to phylogroup A were the predominant strain obtained from the animals used in the study. Four novel sequence types (ST746, ST747, ST748 and ST749) were discovered using the multi locus sequence typing, together with one common ST357 in the gastrointestinal tract, liver and, the trachea and lung. Serotyping demonstrated that these commensal E. coli strains were non-Shiga toxin-producers. Phenotypic and genotypic analyses of extended spectrum beta lactamases were also negative.

Conclusions: These findings implied that the E. coli strains recovered from the laboratory rodents were truly commensal in nature. Further study is required to investigate the possible influence of gender on the susceptibility of hosts to E. coli colonization in laboratory rodents.

Keywords: Escherichia coli, Commensal, Malaysia, Rodent

\section{Background}

Escherichia coli, a non-sporulating, Gram-negative, rodshaped aerobe, is a commensal in the intestines of vertebrates (Duriez et al. 2001; Escobar-Páramo et al. 2006; Jaureguy et al. 2008; Tenaillon et al. 2010; Schmidt et al. 2015), present in various habitats ranging from deserts to the arctic (Escobar-Páramo et al. 2006). This bacteria species also thrives in water environments, with half of the E. coli population estimated to reside in these secondary habitats (Tenaillon et al. 2010), facilitating anthropogenic transmission from species to species through water and food ingestion (Escobar-Páramo et al. 2006). These characteristics suggest that $E$. coli is capable of colonizing and spreading in different environments (Escobar-Páramo

\footnotetext{
*Correspondence: sazaly@um.edu.my

1 Tropical Infectious Diseases Research and Education Centre, Faculty of Medicine, University of Malaya, 50603 Kuala Lumpur, Malaysia Full list of author information is available at the end of the article
}

et al. 2006). Pathogenic strains of $E$. coli can cause serious infections including, intestinal and extraintestinal diseases in humans (Tenaillon et al. 2010). Previous studies have suggested that commensal strains of $E$. coli may be the precursor to the pathogenic E. coli strains following acquisition of virulence genes (Duriez et al. 2001), potentially implicating commensal E. coli as the natural reservoir of pathogenic strains (Schmidt et al. 2015). Enhancement of pathogenicity through random point mutations or acquisition of chromosomal virulence operons were proposed to be methods by which commensal E. coli can become pathogenic (Duriez et al. 2001), and this was possible with $E$. coli having a clonal structure (Tenaillon et al. 2010; Smati et al. 2015) coupled with a high level of genome plasticity.

Phylogenetic analyses have greatly aided our understanding of the genetic structure within the E. coli species by the assignment of at least seven phylogroups 
belonging to $E$. coli sensu stricto (A, B1, B2, C, D, E and F) (Clermont et al. 2013) and the multi locus sequence typing (Jaureguy et al. 2008). Serotyping via molecular typing of the $\mathrm{O}$-antigens could also assist in the study of the genetic structure of E. coli (Sánchez et al. 2015). These methods are powerful tools for determining the phylogenetic relationships among the different $E$. coli lineages and could possibly provide greater understanding of the factors that shape the genetic structure of $E$. coli (Jaureguy et al. 2008; Clermont et al. 2013). However, the vast majority of research focus have been biased towards human E. coli strains. Laboratory rodents have been pivotal as a model for the study of the complex physiological system of mammals. They have contributed immensely to our understanding of the various human diseases, enhancing our knowledge to find effective treatments for these diseases (Jacoby and Lindsey 1998). However, these rodents are also inherently susceptible to numerous known bacterial, fungal and viral diseases (Jacoby and Lindsey 1998). Some of these infectious agents cause subclinical and unnoticeable infections that could nevertheless, adversely affect biological experimental data (Jacoby and Lindsey 1998; Loong et al. 2016).

Animal husbandry practices can potentially alter the composition of laboratory rodent microbiota, affecting experimental outcomes by introducing variables into the animals (Ma et al. 2012). The gastrointestinal microbiota are associated with the regulation of innate immunity and pathogen recognition receptors (Lee et al. 2011). Accordingly, the microbiota has been shown to modulate pro- and anti-inflammatory immune responses in mice models of multiple sclerosis (Lee et al. 2011) and type-1 diabetes (Burrows et al. 2015), suggesting that perturbation of the fragile equilibrium within the gastrointestinal tract impacts the pathologies of mice disease models. Upsetting of the normal microbiota by dysbiosis, abolishes colonization resistance, disrupts homeostasis and increases the host's susceptibility to diseases (Ma et al. 2012). The successful mice intestine colonization by Campylobacter jejuni after antibiotics elimination of the gastrointestinal microbiota (O'Laughlin et al. 2015) demonstrated the effect of the abolishment of colonization resistance. A recent survey in 21 different German animal facilities found that shed skin or dust particles carried by animals, care takers or scientists influenced the gastrointestinal microbiota of experimental mice (Rausch et al. 2016), potentially affecting experimental outcomes. Fluctuations in the laboratory rodent microbiota as a result of external stimuli has to be reduced or eliminated to ensure consistency and reproducibility of experimental results. Therefore, surveillance of commensal E. coli populations in laboratory rodents could provide an indication of possible alteration of the normal gastrointestinal microbiota.
As E. coli is widely used as an indicator of antimicrobial selection pressure (Grønvold et al. 2010) and newly introduced bacteria species may transfer antimicrobial resistant determinants to commensal species (Schmidt et al. 2015), assessment of the genetic characteristics of commensal E. coli would provide information on the virulence status in the animals housed in the animal experimental facility. The occurrence of horizontal gene transfer of beta-lactamases among $E$. coli (Jaureguy et al. 2008; Koga et al. 2015) and the reverse transfer of antimicrobial resistance from humans to animals (Grall et al. 2015) could transpire as a result of the close interaction between scientists (humans) and laboratory rodents (animals). Hence, determination of antimicrobial resistance at the genetic level is important for understanding and limit antimicrobial resistance. The aims of this study were to determine the genetic structure of $E$. coli strains from the organs of otherwise healthy laboratory rodents to establish a background for further studies on pathogenic strains and to analyze the antimicrobial resistance profiles of the isolated E.coli strains.

\section{Methods}

\section{Isolation of E. coli}

A total of 67 randomly selected healthy rodents (mice; $\mathrm{n}=38$ and rats; $\mathrm{n}=29$ ) aged between 6 and 12 weeks old, housed in the Animal Experimental Unit, Faculty of Medicine, University of Malaya during March 2014-April 2015, were assessed as part of the unit's rodents health monitoring program (Table 1). The sex of the rodents were distributed quite proportionately between males $(\mathrm{n}=32)$ and females $(\mathrm{n}=35)$ (Table 1$)$. Healthy mice were euthanized and serologically assayed for selected pathogens according to previously published protocols (Loong et al. 2016). The rats however, were anaesthetized with Ketamine $(50 \mathrm{mg} / \mathrm{kg}$ ) and Xylazine $(5 \mathrm{mg} / \mathrm{kg}$ ) prior to blood collection through cardiac puncture. The collected rat blood was tested by serology (SMART-SPOT Sentinel Panel Test, Rat Sera, Biotech Trading Partners, USA) for the following agents; Kilham rat virus,

\begin{tabular}{lcc}
$\begin{array}{l}\text { Table } \mathbf{1} \text { Breed of rodents assessed between } \\
\text { and April } \mathbf{2 0 1 5}\end{array}$ & March $\mathbf{2 0 1 4}$ \\
\hline Breed & 10 & Female (n) \\
\hline Sprague-Dawley rat & 3 & 10 \\
Wistar Kyoto rat & 2 & 3 \\
Spontaneously hypertensive rat & 14 & 1 \\
ICR mouse & 3 & 15 \\
BALB/c mouse & 0 & 3 \\
C57BL/6 mouse & 67 & 3 \\
Total &
\end{tabular}


Mycoplasma pulmonis, pneumonia virus of mice, rat coronavirus, respiratory enteric orphan virus, rat parvovirus, Sendai virus and Theiler's murine encephalomyelitis virus. Following that, the rats were euthanized by overdosing with the same drugs at three times the anaesthetic dosage. Rodents were housed in open cages and other detailed procedures were described in a previous study (Loong et al. 2016). Selected rodent organs (gastrointestinal tract, liver and, the trachea and lung) were harvested, homogenized and cultured onto MacConkey agar. Organs from every rodent were cultured onto individual agar plate. Cultured bacterial strains suspected to be $E$. coli were identified using Gram staining, microscopy and 16S rDNA sequencing (Misbah et al. 2005). One strain was selected from each suspected culture plates after $24 \mathrm{~h}$ incubation at $37{ }^{\circ} \mathrm{C}$ for this study.

\section{Multi locus sequence typing (MLST) and phylogroup classification}

Commensal E. coli strains were subjected to MLST (referred to as the Pasteur scheme), where the internal portions of eight housekeeping genes $(\operatorname{din} B, i c d A, p a b B$, polB, putP, $\operatorname{trp} A, \operatorname{trp} B$ and uidA) were sequenced and compared with the database at http://bigsdb.web.pasteur. fr/ecoli/ecoli.html (Jaureguy et al. 2008). A specific allele number was assigned to each distinct sequence within a locus and a specific sequence type (ST) was assigned to each distinct combination of alleles. A novel allele number was assigned to sequences that could not be found in the database, subsequently establishing a novel ST. For phylogroup classification, $E$. coli strains were designated into the phylogroups; A, B1, B2, C, D, E, or F, according to a previous study (Clermont et al. 2013). This quadruplex PCR method differentiates the $E$. coli phylogroups by nucleic acid amplification of the $\operatorname{arp} A$, chuA and $y j a A$ genes, and the DNA fragment, TspE4.C2.

Identification of Shiga toxin-producing $E$. coli serotypes E. coli serogroups O5, O15, O26, O45, O55, O76, O91, O103, O104, O111, O113, O118, O121, O123, O128, O145, O146, O157, O165, O172 and O177 are the O-antigen types of the most clinically significant Shiga toxin-producing E. coli serotypes. These 21 serotypes were identified using three serotype-specific multiplex PCR assays, and it can also detect the respective $\mathrm{O}$-antigens even when they are not expressed by the $E$. coli strains (Sánchez et al. 2015).

\section{Extended spectrum beta lactamases (ESBL) phenotypic confirmatory method}

All commensal E. coli strains were cultured onto Mueller-Hinton agar after adjustment to $0.5 \mathrm{McF}$ arland standards. Antimicrobial discs containing cefotaxime $(30 \mu \mathrm{g})$, cefotaxime/clavulanic acid $(30 / 10 \mu \mathrm{g})$, ceftazidime $(30 \mu \mathrm{g})$ and ceftazidime/clavulanic acid $(30 / 10 \mu \mathrm{g})$ were placed onto the agar plate so that the distances between them are approximately twice the radius of the inhibition zones, and the plate was incubated overnight at $35{ }^{\circ} \mathrm{C}$. ESBL activity was indicated when $\mathrm{a} \geq 5 \mathrm{~mm}$ increase in the inhibition zone diameter around either cephalosporin disc in combination with clavulanic acid as compared to the inhibition zone diameter of the cephalosporin when tested alone (CLSI 2012). The E. coli strain ATCC 25922 and Klebsiella pneumoniae strain ATCC 700603 were used as controls as per CLSI recommendation.

\section{Detection of genes encoding for ESBL}

Five multiplex and one singleplex PCRs were employed in this study to detect genes encoding important betalactamases; one CTX-M multiplex PCR including groups 1, 2, 8, 9 and 25 (Woodford et al. 2006); one TEM/SHV/ OXA-1-like multiplex PCR (Dallenne et al. 2010); one plasmid-mediated AmpC gene multiplex PCR including ACC, FOX, MOX, DHA, CIT and EBC (Dallenne et al. 2010); one VEB/GES/PER multiplex PCR (Dallenne et al. 2010); one VIM/IMP/KPC multiplex PCR (Dallenne et al. 2010); and one OXA-48-like singleplex PCR (Dallenne et al. 2010). In addition, the New Delhi metallo-betalactamase, NDM-1 was detected using PCR as previously described (Peirano et al. 2011).

\section{Detection of $E$. coli from the animal housing environment, rodent feed and drinking water}

Testing for the presence of $E$. coli in the animal housing environment was performed by taking swabs from the tabletop, tap, trolley and wash basin in the room housing the rodents. Sterile, irradiated rodent feed (\#1320-Maintenance diet for rats and mice, Altromin International, Germany) was vortexed in tubes containing nuclease-free water. Following that, the swabs, the vortexed feed and the autoclaved drinking water were cultured onto MacConkey agar and incubated at $37^{\circ} \mathrm{C}$ under aerobic condition for $24 \mathrm{~h}$. One colony resembling the morphological appearance of $E$. coli was selected from each positive plate and the E. coli phylogroup was determined. Additionally, detection of genes encoding for ESBL was also performed.

\section{Results}

In the study period of 14 months (March 2014-April 2015), we randomly selected and examined a total of 67 healthy rodents of various breeds, housed in the AAALAC-certified facility at the Animal Experimental Unit, University of Malaya. This was performed as part of the health monitoring program to ensure that the rodents are free of specific pathogens that could affect 
scientific experiments. A total of 13 commensal E. coli strains were recovered on MacConkey agars, from the gastrointestinal tract, liver and, the trachea and lung of laboratory rodents (Table 2). Accession numbers for the partial $16 \mathrm{~S}$ rDNA sequences of the 13 commensal $E$. coli strains include, LT009415-LT009427. All 13 commensal E. coli strains in this study were recovered from female rodents. These 13 strains were cultured from 10 different, individual female rodents and, we did not manage to isolate $E$. coli from the remaining 54 rodents. E. coli was isolated mainly from the gastrointestinal tract $(\mathrm{n}=6)$ and, the trachea and lung $(n=5)$, with the remaining 2 strains from the liver. A majority of the commensal $E$. coli strains were found in ICR mice $(\mathrm{n}=8)$. Meanwhile, C57BL/6 mice harbored 2 strains and, the BALB/c mouse, Sprague-Dawley and Wistar Kyoto rats, each harbored 1 strain. Two E. coli strains were isolated from the different organs of 3 individual ICR mice. Strains UM-AEU197 and 198 were isolated from the liver and, the trachea and lung of ICR mouse \#1, respectively. Strains UM-AEU202 and 203 were isolated respectively, from the liver and, the trachea and lung of ICR mouse \#2. The trachea and lung, and the gastrointestinal tract of ICR mouse \#3 sheltered strains UM-AEU213 and 214, respectively (Table 2).

Analysis of the commensal E. coli strains by MLST revealed 13 strains with 4 novel and 1 common STs (Table 2). The E. coli strains recovered from ICR mice, were designated into ST748 and ST749. Strains recovered from Sprague-Dawley and Wistar Kyoto rats were designated into ST746 and ST747, respectively. In addition, ST747 was also found in a C57BL/6 mouse. The sole common E. coli ST357 was recovered from the gastrointestinal tract of another C57BL/6 mouse. The gastrointestinal tract was found to harbor all 5 different STs (ST357, ST746, ST747, ST748 and ST749) in this study. The liver and, the trachea and lung however, only harbored ST749 and it was also the most frequent ST encountered. Analysis into the phylogroups of the commensal E. coli strains by the quadruplex PCR method revealed that all 13 strains belonged to phylogroup A (Table 2). The alignments of all eight $E$. coli MLST loci relative to the respective reference Allele 1 are shown in Additional files 1, 2, $3,4,5,6,7$ and 8 .

The multiplex PCRs to detect the most common clinically significant Shiga toxin-producing E. coli serotypes showed that all 13 commensal E. coli strains in this study did not belong to any of the serotypes (Additional file 9). Similarly, we observed negative ESBL activity in the 13 commensal E. coli strains when tested using the ESBL phenotypic confirmatory method (Table 3). Negative ESBL activity however, does not necessarily mean that the $E$. coli strain is negative for ESBL encoding genes, therefore a series of nucleic acid amplifications for the detection of ESBL encoding genes was performed. However, no ESBL encoding gene was amplified from the commensal E. coli strains recovered in our study (Additional files 10, 11, 12).

Testing of the animal housing environment, feed and drinking water of the laboratory rodents for the presence of $E$. coli showed that only the wash basin sample resulted in positive $E$. coli culture. The cultured $E$. coli strain belonged to phylogroup B1 and no ESBL genes

Table 2 Genetic characteristics of commensal $E$. coli strains from rodents in the animal experimental unit

\begin{tabular}{|c|c|c|c|c|c|c|c|c|c|c|c|c|c|}
\hline \multirow[t]{2}{*}{ Strain } & \multirow[t]{2}{*}{ Breed $^{a}$} & \multirow[t]{2}{*}{ Organ $^{\mathbf{b}}$} & \multirow[t]{2}{*}{ Sex } & \multirow{2}{*}{$\begin{array}{l}\text { Phylogenetic } \\
\text { group }\end{array}$} & \multicolumn{8}{|c|}{ MLST locus } & \multirow[t]{2}{*}{$\overline{\mathrm{ST}}$} \\
\hline & & & & & $\operatorname{din} B$ & $i c d A$ & $p a b B$ & polB & putP & $\operatorname{trp} A$ & $\operatorname{trpB}$ & uidA & \\
\hline UM-AEU015 & SD & GIT & $F$ & A & 132 & 65 & 3 & 10 & 26 & 53 & 4 & 65 & 746 \\
\hline UM-AEU018 & C57BL/6 & GIT & $F$ & A & 132 & 65 & 3 & 10 & 26 & 53 & 4 & 65 & 746 \\
\hline UM-AEU021 & C57BL/6 & GIT & $F$ & A & 5 & 47 & 4 & 10 & 26 & 1 & 4 & 2 & 357 \\
\hline UM-AEU116 & WKY & GIT & $F$ & A & 133 & 41 & 47 & 169 & 178 & 23 & 20 & 164 & 747 \\
\hline UM-AEU131 & ICR & GIT & $\mathrm{F}$ & A & 25 & 65 & 3 & 10 & 180 & 53 & 4 & 65 & 748 \\
\hline UM-AEU140 & $\mathrm{BALB} / \mathrm{C}$ & T\&L & F & A & 25 & 65 & 48 & 10 & 179 & 8 & 2 & 2 & 749 \\
\hline UM-AEU197 & ICR & Liv & $F$ & A & 25 & 65 & 48 & 10 & 179 & 8 & 2 & 2 & 749 \\
\hline UM-AEU198 & ICR & T\&L & $F$ & A & 25 & 65 & 48 & 10 & 179 & 8 & 2 & 2 & 749 \\
\hline UM-AEU202 & ICR & Liv & $F$ & A & 25 & 65 & 48 & 10 & 179 & 8 & 2 & 2 & 749 \\
\hline UM-AEU203 & ICR & T\&L & $F$ & A & 25 & 65 & 48 & 10 & 179 & 8 & 2 & 2 & 749 \\
\hline UM-AEU208 & ICR & T\&L & $F$ & A & 25 & 65 & 48 & 10 & 179 & 8 & 2 & 2 & 749 \\
\hline UM-AEU213 & ICR & T\&L & $F$ & A & 25 & 65 & 48 & 10 & 179 & 8 & 2 & 2 & 749 \\
\hline UM-AEU214 & ICR & GIT & $F$ & A & 25 & 65 & 48 & 10 & 179 & 8 & 2 & 2 & 749 \\
\hline
\end{tabular}

a Breed; SD Sprague-Dawley rat, WKY Wistar Kyoto rat, BALB/C BALB/c mouse, C57BL/6 C57BL/6 mouse, ICR ICR mouse

b Organ; GIT gastrointestinal tract, $T \& L$ trachea and lungs, Liv liver

c Sex; $F$ female 
Table 3 Prevalence of ESBL producers among $E$. coli Strains from rodents in the animal experimental unit

\begin{tabular}{|c|c|c|c|c|c|}
\hline \multirow[t]{2}{*}{ Strains } & \multicolumn{4}{|c|}{ Zone diameter (mm) } & \multirow[t]{2}{*}{ ESBL producing organism ${ }^{\mathbf{b}}$} \\
\hline & CTX ${ }^{a}$ & $C T X+C A^{a}$ & $C A Z^{a}$ & $C A Z+C A^{a}$ & \\
\hline K. pneumoniae ATCC 700603 & 6 & 28 & 7 & 20 & + \\
\hline E. coli ATCC 25922 & 15 & 15 & 16 & 16 & - \\
\hline UM-AEU015 & 14 & 14 & 14 & 14 & - \\
\hline UM-AEU018 & 13 & 13 & 15 & 15 & - \\
\hline UM-AEU021 & 16 & 16 & 16 & 16 & - \\
\hline UM-AEU116 & 15 & 16 & 15 & 16 & - \\
\hline UM-AEU131 & 13 & 13 & 14 & 14 & - \\
\hline UM-AEU140 & 15 & 15 & 15 & 16 & - \\
\hline UM-AEU197 & 14 & 15 & 15 & 16 & - \\
\hline UM-AEU198 & 13 & 14 & 14 & 14 & - \\
\hline UM-AEU202 & 13 & 13 & 13 & 14 & - \\
\hline UM-AEU203 & 15 & 16 & 16 & 17 & - \\
\hline UM-AEU208 & 14 & 14 & 15 & 16 & - \\
\hline UM-AEU213 & 15 & 15 & 16 & 16 & - \\
\hline UM-AEU214 & 14 & 15 & 15 & 15 & - \\
\hline
\end{tabular}

${ }^{a} \mathrm{CTX}=$ cefotaxime, $\mathrm{CTX}+\mathrm{CA}=$ cefotaxime-clavulanic acid, $\mathrm{CAZ}=$ ceftazidime, $\mathrm{CAZ}+\mathrm{CA}=$ ceftazidime-clavulanic acid

${ }^{b}$ ESBL producing organism; + = ESBL producer, $-=$ non-ESBL producer

were detected by PCR. E. coli was not cultured from the other animal housing environment samples (table top, tap and trolley), rodent feed and drinking water (Table 4).

\section{Discussion}

In this study, several key factors involved in influencing the distribution of E. coli populations in laboratory rodents were controlled. MacConkey agar was used for the culture of $E$. coli strains in this study because it has the propensity to culture members of the Enterobacteriaceae and cultured E. coli colonies can be easily distinguished by its morphology and color (Gordon and Cowling 2003). Consequently, we only sampled one $E$. coli strain from each suspected agar plate representing the most dominant strain despite evidence that hosts

Table 4 Prevalence of $E$. coli in the animal housing environment, rodent feed and drinking water

\begin{tabular}{llll}
\hline Sample & $\begin{array}{l}\text { Presence of } \\
E_{\text {. coli }}\end{array}$ & $\begin{array}{l}\text { Phylogenetic } \\
\text { group }^{\mathbf{b}}\end{array}$ & $\begin{array}{l}\text { Presence of } \\
\text { ESBL genes }^{\mathbf{c}}\end{array}$ \\
\hline Table top & - & - & $\mathrm{NP}$ \\
Tap & - & - & $\mathrm{NP}$ \\
Trolley & - & - & $\mathrm{NP}$ \\
Wash basin & + & $\mathrm{B} 1$ & - \\
Rodent feed & - & - & $\mathrm{NP}$ \\
Drinking water & - & - & $\mathrm{NP}$ \\
\hline
\end{tabular}

\footnotetext{
a Presence of $E$. coli; $+=E$. coli present, $-=E$. coli not present

b Phylogenetic group; $\mathrm{B} 1=E$. coli phylogroup $\mathrm{B} 1,-=$ not detected

c Presence of ESBL genes; - = ESBL genes not detected, NP = not performed
}

can be colonized with more than one $E$. coli strain at the same time (Gordon et al. 2005). Even though E. coli is an established colonizer of the mouse gut (Skurnik et al. 2016), translocation to other organs including the liver (Ohsugi et al. 1996) and the lung (Wu et al. 2010) has been reported. Therefore, in our study, we examined the other rodent organs (liver and, the trachea and lung) for the presence of $E$. coli. In agreement with the previous reports (Ohsugi et al. 1996; Wu et al. 2010), we found two E. coli strains, each, from different organs in three individual ICR mice. The rodent skin was scrubbed with antiseptic prior to surgery and the harvesting of rodent organs in the following sequence; firstly, the trachea and lung, secondly, the liver, and lastly, the gastrointestinal tract. New sets of sterile surgical tools were utilized for the harvesting of different organs. These procedures were performed to prevent E. coli cross contamination and translocation was evident with the sighting of similar STs among E. coli strains from different organs of the same individual.

The distribution of E. coli populations in the mammalian host depended on the host habitat, diet, gut anatomy and body mass (Gordon and Cowling 2003). In humans, morphological, physiological and dietary differences among individuals of different sex and age influence the distribution of commensal E. coli populations (Gordon et al. 2005). It was further suggested that the exposure to novel E. coli strains and the health of the animal might contribute to the temporal variability of commensal E. coli populations in animals (Grønvold et al. 
2010). Shifting of the commensal E. coli population in laboratory rodents can alter experimental outcomes by stressing the rodent's microbiota into a state of imbalance (Ma et al. 2012) and this is not desired in any animal experimental facilities. Previous study has reported a lower likelihood for the transmission of bacterial plasmids carrying virulence genes to animals housed separately or in small groups, in comparison to animals raised in cramped and dense conditions (Koga et al. 2015). The rodents in this study were housed at a barrier facility in open cages maintained at a constant room temperature, relative humidity and light:dark cycle (Loong et al. 2016). Standard operating procedures including, the wearing of personal protective equipment, working on one animal at a time in the biosafety cabinet, changing gloves after handling a single animal and performing surgeries in a dedicated room away from breeding facilities (Loong et al. 2016) were followed as per required under AAALAC accreditation to prevent introduction of other bacteria species into the studied cohort. To prevent possible transmission from the animal housing environment, several sites in the room where the rodents were housed (table top, tap, trolley and wash basin), were selected to test for the presence of $E$. coli. Similarly, the rodent's feed and drinking water were also tested for the presence of E. coli. We however, found that only the sample from the wash basin grew $E$. coli belonging to phylogroup B1 with no detectable ESBL genes. The presence of $E$. coli phylogroup B1 only at the wash basin suggests that the strain favors the water environment, agreeing with the earlier report by Ratajczak et al. (2010) who found phylogroup B1 strains to be specifically adapted and persistent in water.

The rodents were fed with diet comprising of products made of soy and corn, equivalent to a herbivorous diet. Identical animal feed was maintained throughout the study without the change of vendor or food variant, and the non-alteration of diet preserves the condition in the gut favoring the dominant E. coli strain (Blyton et al. 2013). Introduction of different diets would disturb the gut ecosystem leading to the colonization of a different $E$. coli strain (Grønvold et al. 2010). Sexually mature healthy rodents (Kempermann et al. 1997; Sengupta 2013) were selected because the dominant commensal E. coli strains would have successfully established stable colonization in the gut flora (Tenaillon et al. 2010) and good health ensured no shifting of host physiological condition (Grønvold et al. 2010), possibly displacing dominant $E$. coli strains in unhealthy animals. The isolation of commensal E. coli only from females in this study suggests that host gender could influence the distribution of $E$. coli and this was similar to human E. coli strains (Gordon et al. 2005), even though the number of rodent strains in this study was relatively small to draw a definite conclusion. In terms of the gut anatomy, E. coli is inclined to flourish in hosts (herbivores and omnivores) with intestinal tracts resembling a microbial fermentation chamber such as a caecum (Gordon and Cowling 2003; Gordon et al. 2005) and all rodents possess a caecum (Gordon and Cowling 2003). In addition, the likelihood of isolating E. coli increases with the body mass of animals (Gordon et al. 2005). This was not the case in our study however, where smaller mice contributed to $>80 \%(11 / 13)$ of the isolated commensal E. coli strains.

Assessment of the E. coli phylogroups categorized the strains isolated from laboratory mice and rats into a single phylogroup A. This finding is largely concordant with other published results on free-range poultry (Koga et al. 2015) and domesticated animals (Tenaillon et al. 2010), where the animals were not housed in dense conditions. Phylogroup B1 strains are best adapted in animals, followed by phylogroup A (Tenaillon et al. 2010), suggesting consistency in our results. The population diversity of $E$. coli due to host diet has been reported with phylogroup A strains predominating in carnivores and omnivores (Escobar-Páramo et al. 2006). Rodents by nature are omnivores and although they were fed a herbivorous diet in this study, it did not change the genetic structure of commensal E. coli strains, suggesting the effect of nutrients on the selection of strains. Perhaps the threshold concentration of nutrients could not be achieved from a herbivorous diet, hence phylogroups associated to herbivores could not succeed in colonization (Gordon et al. 2005). The probability of discovering phylogroup A strains is also higher in female humans (Gordon et al. 2005), similar to the findings in laboratory rodents in this study. Another possible explanation for this finding could be that sex hormones may modulate host physiological conditions and it has been demonstrated in humans that sex hormones provoke metabolic changes that shape the intestinal microbiota (Koren et al. 2012). The association between the female gender and $E$. coli colonization suggests laboratory rodents to be a good model for studying the impact of sex hormones on the colonization of other commensal bacteria species. Gordon and Cowling (2003) also reported from their survey of the distribution of $E$. coli in Australian vertebrates that phylogroup A can be recovered from any vertebrate group, however this phylogroup is also responsible for causing intestinal infections in mammals. The finding of a different phylogroup B1 from the wash basin further supports our suggestion that the strains recovered from the laboratory rodents were true commensals and transmission from the animal housing environment, feed or water did not occur. The absence of ESBL genes in the $E$. coli strain isolated from the wash basin indicated that the rodent housing facility 
was not tainted by any clinical bacterial strains carrying resistance genes.

The discovery of 4 novel STs (ST746-ST749) on top of one common ST (ST357) from a relatively small number of specimens using MLST, signified that the rodent's microbiota is more diverse than the human's (Smati et al. 2015). Our findings revealed a distinct demarcation of STs for laboratory rodents, with ST357, ST748 and ST749 found in mice, and ST747 in rats (Table 2). This could be explained by the genetic variability among different rodent breeds (Wu et al. 2001) that predisposes them to having different physiological responses (Gibney et al. 2010) and, hormone changes are known to affect bacteria colonization in the host (Blyton et al. 2013). Isolation of ST746 from both mouse and rat suggest that this ST could have adapted to the physiology of different rodent breeds. Based on ST similarities in the different $E$. coli strains cultured from the liver and, the trachea and lung specimens denote that the rodent body is frequently exposed to commensal bacteria from the gastrointestinal tract (Wu et al. 2001). It has been widely accepted that $E$. coli phylogroups are non-randomly distributed among mammalian hosts (Gordon et al. 2005; Clermont et al. 2013) and all of these findings imply that phylogroup A strains establish an ecological niche in laboratory rodents.

Serotyping of the isolated commensal E. coli strains using multiplex PCR assays revealed that all the rodent strains were negative for the 21 most clinically relevant Shiga toxin-producing E. coli. All commensal E. coli strains were also negative for ESBL production and genes encoding beta lactamases. This was not surprising, considering that the rodents were housed in proper AAALAC accredited animal housing facility. The level of hygiene in the human population was noted as one of the main factors for the significant shift in the proportions of E. coli strains in France (Tenaillon et al. 2010) and an increased level of antimicrobial resistance in $E$. coli isolated from animals was observed with an increase density of human population living in close proximity to these animals (Skurnik et al. 2016). Furthermore, we could not culture E. coli from a large number of our specimens, suggesting that the bacteria density was very low in the organs and low bacteria density would restrict the occurrence of horizontal resistance gene transfer (Skurnik et al. 2016). The low bacteria density and the absence of antimicrobial resistance genes in commensal E. coli strains of laboratory rodents is consistent with the hypothesis that strains with more virulence genes are likely to command better survival fitness and persist longer in the gut (Blyton et al. 2013). One limitation of this study was the relatively small number of isolated strains and the study was performed in only one animal experimental facility. It is possible that $E$. coli strains from other animal species and other animal experimental facilities would differ.

\section{Conclusions}

This study found that the commensal E. coli strains isolated from different rodent breeds housed in the Animal Experimental Unit, Faculty of Medicine, University of Malaya were categorized as members of phylogroup A. Four novel sequence types were uncovered using MLST, displaying the broad diversity of commensal $E$. coli strains from laboratory rodents. As expected, the isolated E. coli strains did not possess antibiotic resistance genes and, were not Shiga toxin and extended spectrum beta lactamase producers. We propose that animal experimental facilities should initiate surveillance of commensal E. coli strains for the determination of phylogroups and the presence of ESBL genes, to monitor for events that could lead to dysbiosis and distortion of animal experimental data. Taken together, the absence of antimicrobial resistance and virulence genes in the E. coli cohort of this study indicate the true commensal nature of these strains. To the best of our knowledge, this study is also the first to analyze the genetic structure of commensal $E$. coli in laboratory rodents.

\section{Additional files}

Additional file 1. Sequence alignments of E. coli MLST dinB loci. Allelic profiles of commensal E. coli were compared against Allele 1 (Ref-dinB) and positions at which differences were found relative to Allele 1, were noted above the reference allele. Each position represents point mutations relative to the reference allele. Nucleotide differences were displayed whereas black dots represent nucleotides similar to the reference allele.

Additional file 2. Sequence alignments of E. coli MLST icdA loci. Allelic profiles of commensal E. coli were compared against Allele 1 (Ref-icdA) and positions at which differences were found relative to Allele 1, were noted above the reference allele. Each position represents point mutations relative to the reference allele. Nucleotide differences were displayed whereas black dots represent nucleotides similar to the reference allele.

Additional file 3. Sequence alignments of E. coli MLST pabB loci. Allelic profiles of commensal E. coli were compared against Allele 1 (Ref-pabB) and positions at which differences were found relative to Allele 1, were noted above the reference allele. Each position represents point mutations relative to the reference allele. Nucleotide differences were displayed whereas black dots represent nucleotides similar to the reference allele.

Additional file 4. Sequence alignments of E. coli MLST polB loci. Allelic profiles of commensal E. coli were compared against Allele 1 (Ref-polB) and positions at which differences were found relative to Allele 1, were noted above the reference allele. Each position represents point mutations relative to the reference allele. Nucleotide differences were displayed whereas black dots represent nucleotides similar to the reference allele.

Additional file 5. Sequence alignments of E. coli MLST putP loci. Allelic profiles of commensal E. coli were compared against Allele 1 (Ref-putP) and positions at which differences were found relative to Allele 1, were noted above the reference allele. Each position represents point mutations relative to the reference allele. Nucleotide differences were displayed whereas black dots represent nucleotides similar to the reference allele. Hyphens represent deletion at that particular allele. 
Additional file 6. Sequence alignments of E. coli MLST trpA loci. Allelic profiles of commensal E. coli were compared against Allele 1 (Ref-trpA) and positions at which differences were found relative to Allele 1, were noted above the reference allele. Each position represents point mutations relative to the reference allele. Nucleotide differences were displayed whereas black dots represent nucleotides similar to the reference allele.

Additional file 7. Sequence alignments of E. coli MLST trpB loci. Allelic profiles of commensal E. coli were compared against Allele 1 (Ref-trpB) and positions at which differences were found relative to Allele 1, were noted above the reference allele. Each position represents point mutations relative to the reference allele. Nucleotide differences were displayed whereas black dots represent nucleotides similar to the reference allele.

Additional file 8. Sequence alignments of E. coli MLST uidA loci. Allelic profiles of commensal E. coli were compared against Allele 1 (Ref-uidA) and positions at which differences were found relative to Allele 1, were noted above the reference allele. Each position represents point mutations relative to the reference allele. Nucleotide differences were displayed whereas black dots represent nucleotides similar to the reference allele.

Additional file 9. Gel electrophoresis images Shiga toxin-producing E. coli serotypes. A) Multiplex PCR \#1. Positive control = O103; O5, O26 O91, 0111, 0121 and 0145 positive controls were not included as we do not have strains encoding those genes. B) Multiplex PCR \#2. Positive control = O76; O45, O55, O113, O128, O146 and 0177 positive controls were not included as we do not have strains encoding those genes. C) Multiplex PCR \#3. Positive control = 0118; 015, 0104, O123, 0157, 0165 and 0172 positive controls were not included as we do not have strains encoding those genes. $-=$ negative control, $1=U M-A E U 015,2=U M-$ AEU018, $3=$ UM-AEU021, $4=$ UM-AEU116, $5=$ UM-AEU131, $6=$ UMAEU140, $7=$ UM-AEU197, $8=$ UM-AEU198, $9=$ UM-AEU202, $10=U M-$ AEU203, $11=$ UM-AEU208, $12=$ UM-AEU213, $13=$ UM-AEU214

Additional file 10. Gel electrophoresis images of genes encoding for ESBL. A) CTX-M multiplex PCR. Positive control = CTX-M group 9; other CTX-M groups 1, 2, 8 and 25 positive controls were not included as we do not have strains encoding those genes. B) TEM/SHV/OXA-1-like multiplex PCR. Positive control = TEM and SHV; OXA-1-like positive control was not included as we do not have strains encoding that gene. - = negative control, 1 = UM-AEU015, 2 = UM-AEU018, $3=$ UM-AEU021, $4=$ UM-AEU116, $5=$ UM-AEU131, 6 = UM-AEU140, 7 = UM-AEU197, 8 = UM-AEU198, $9=$ UM-AEU202, 10 = UM-AEU203, $11=$ UM-AEU208, $12=$ UM-AEU213, $13=U M-A E U 214$

Additional file 11. Gel electrophoresis images of genes encoding for ESBL. A) Plasmid-mediated AmpC gene multiplex PCR. Positive control = LAT-1; ACC, FOX, MOX, DHA and EBC positive controls were not included as we do not have strains encoding those genes. B) VEB/GES/ PER multiplex PCR. Positive control = GES; VEB and PER positive controls were not included as we do not have strains encoding those genes. - = negative control, 1 = UM-AEU015, 2 = UM-AEU018, 3 = UM-AEU021, $4=$ UM-AEU116, $5=$ UM-AEU131, $6=$ UM-AEU140, $7=$ UM-AEU197, $8=U M-A E U 198,9=U M-A E U 202,10=U M-A E U 203,11=U M-A E U 208$, $12=U M-A E U 213,13=U M-A E U 214$

Additional file 12. Gel electrophoresis images of genes encoding for ESBL. A) VIM/IMP/KPC multiplex PCR. Positive control = IMP; VIM and KPC positive controls were not included as we do not have strains encoding those genes. B) OXA-48-like PCR. Positive control = OXA-48. - = negative control, 1 = UM-AEU015, 2 = UM-AEU018, $3=$ UM-AEU021, $4=$ UMAEU116, $5=$ UM-AEU131, $6=$ UM-AEU140, $7=$ UM-AEU197, $8=$ UMAEU198, $9=$ UM-AEU202, $10=$ UM-AEU203, $11=U M-A E U 208,12=U M-$ AEU213, $13=U M-A E U 214$. Since we do not have positive control for NDM-1, the gel electrophoresis image was not presented.

\section{Authors' contributions}

SKL conceived, designed, performed the study and analyzed the data. NHM and NAACMS were involved in laboratory experiments and data analyses. HAMW, SAAG and PFW were involved in caring of the animals and performing animal surgeries. SAB was involved in experimental design and supervised data analyses. SKL and SAB drafted the manuscript. All authors read and approved the final manuscript.

\section{Author details}

${ }^{1}$ Tropical Infectious Diseases Research and Education Centre, Faculty of Medicine, University of Malaya, 50603 Kuala Lumpur, Malaysia. ${ }^{2}$ Animal Experimental Unit, Faculty of Medicine, University of Malaya, 50603 Kuala Lumpur, Malaysia. ${ }^{3}$ Department of Pharmacology, Faculty of Medicine, University of Malaya, 50603 Kuala Lumpur, Malaysia. ${ }^{4}$ Department of Medical Microbiology, Faculty of Medicine, University of Malaya, 50603 Kuala Lumpur, Malaysia.

\section{Acknowledgements}

The authors thank Dr. Cindy Shuan-Ju Teh for providing the E. coli clinical strains as controls in the PCR assays. We also thank the team of curators of the Institut Pasteur MLST and whole genome MLST databases for curating the data and make them publicly available at http://bigsdb.web.pasteur.fr/.

\section{Competing interests}

The authors declare that they have no competing interests.

\section{Ethics approval and consent to participate}

This study and all animal handling procedures were approved by the Institutional Animal Care and Use Committee, University of Malaya (Ref. No. 2013-11-12/AEU/B/WPF).

\section{Funding}

This study was supported in part by the UM Research University Grant-Tropical Infectious Diseases Programme (RU016-2015), the High Impact Research-Ministry of Higher Education Grant (E000013-20001) and the University of Malaya Research Grant (RP016C-14AFR). The funders had no role in the study design, data collection and analysis, interpretation of data, decision to publish, or preparation of the manuscript.

Received: 24 April 2016 Accepted: 2 July 2016

Published online: 11 July 2016

\section{References}

Blyton MD, Banks SC, Peakall R, Gordon DM (2013) High temporal variability in commensal Escherichia coli strain communities of a herbivorous marsupial. Environ Microbiol 15:2162-2172

Burrows MP, Volchkov P, Kobayashi KS, Chervonsky AV (2015) Microbiota regulates type 1 diabetes through toll-like receptors. Proc Natl Acad Sci USA 112:9973-9977

Clermont O, Christenson JK, Denamur E, Gordon DM (2013) The Clermont Escherichia coli phylo-typing method revisited: improvement of specificity and detection of new phylo-groups. Environ Microbiol Rep 5:58-65

Clinical and Laboratory Standards Institute (CLSI) (2012) Performance standards for antimicrobial susceptibility testing; 22nd informational supplement, CLSI document M100-S22. Wayne, Pennsylvania

Dallenne C, Da Costa A, Decré D, Favier C, Arlet G (2010) Development of a set of multiplex PCR assays for the detection of genes encoding important $\beta$-lactamases in Enterobacteriaceae. J Antimicrob Chemother 65:490-495

Duriez P, Clermont O, Bonacorsi S, Bingen E, Chaventré A, Elion J, Picard B, Denamur E (2001) Commensal Escherichia coli isolates are phylogenetically distributed among geographically distinct human populations. Microbiology 147:1671-1676

Escobar-Páramo P, Le Menac'h A, Le Gall T, Amorin C, Gouriou S, Picard B, Skurnik D, Denamur E (2006) Identification of forces shaping the commensal Escherichia coli genetic structure by comparing animal and human isolates. Environ Microbiol 8:1975-1984

Gibney SM, Gosselin RD, Dinan TG, Cryan JF (2010) Colorectal distensioninduced prefrontal cortex activation in the Wistar-Kyoto rat: implications for irritable bowel syndrome. Neuroscience 165:675-683

Gordon DM, Cowling A (2003) The distribution and genetic structure of Escherichia coli in Australian vertebrates: host and geographic effects. Microbiology 149:3575-3586 
Gordon DM, Stern SE, Collignon PJ (2005) Influence of the sex of human hosts on the distribution of Escherichia coli ECOR groups and virulence traits. Microbiology 151:15-23

Grall N, Barraud O, Wieder I, Hua A, Perrier M, Babosan A, Gaschet M, Clermont O, Denamur E, Catzeflis F, Decré D, Ploy MC, Andremont A (2015) Lack of dissemination of acquired resistance to $\beta$-lactams in small wild mammals around an isolated village in the Amazonian forest. Environ Microbiol Rep 7:698-708

Grønvold AM, L'abée-Lund TM, Sørum H, Skancke E, Yannarell AC, Mackie RI (2010) Changes in fecal microbiota of healthy dogs administered amoxicillin. FEMS Microbiol Ecol 71:313-326

Jacoby RO, Lindsey JR (1998) Risks of infection among laboratory rats and mice at major biomedical research institutions. ILAR J 39:266-271

Jaureguy F, Landraud L, Passet V, Diancourt L, Frapy E, Guigon G, Carbonnelle E, Lortholary O, Clermont O, Denamur E, Picard B, Nassif X, Brisse S (2008) Phylogenetic and genomic diversity of human bacteremic Escherichia coli strains. BMC Genom 9:560

Kempermann G, Kuhn HG, Gage FH (1997) Genetic influence on neurogenesis in the dentate gyrus of adult mice. Proc Natl Acad Sci USA 94:10409-10414

Koga VL, Scandorieiro S, Vespero EC, Oba A, de Brito BG, de Brito KC, Nakazato G, Kobayashi RK (2015) Comparison of antibiotic resistance and virulence factors among Escherichia coli isolated from conventional and free-range poultry. Biomed Res Int 2015:618752

Koren O, Goodrich JK, Cullender TC, Spor A, Laitinen K, Bäckhed HK, Gonzalez A, Werner JJ, Angenent LT, Knight R, Bäckhed F, Isolauri E, Salminen S, Ley RE (2012) Host remodeling of the gut microbiome and metabolic changes during pregnancy. Cell 150:470-480

Lee YK, Menezes JS, Umesaki Y, Mazmanian SK (2011) Proinflammatory T-cell responses to gut microbiota promote experimental autoimmune encephalomyelitis. Proc Natl Acad Sci USA 108:4615-4622

Loong SK, Mahfodz NH, Mohamad Wali HA, Talib SA, Ahmad Nasrah SN, Wong PF, AbuBakar S (2016) Molecular and antimicrobial analyses of non-classical Bordetella isolated from a laboratory mouse. J Vet Med Sci 78:715-717

Ma BW, Bokulich NA, Castillo PA, Kananurak A, Underwood MA, Mills DA, Bevins CL (2012) Routine habitat change: a source of unrecognized transient alteration of intestinal microbiota in laboratory mice. PLoS One 7:e47416

Misbah S, Hassan H, Yusof MY, Hanifah YA, AbuBakar S (2005) Genomic species identification of Acinetobacter of clinical isolates by 165 rDNA sequencing. Singap Med J 46:461-464

O'Laughlin JL, Samuelson DR, Braundmeier-Fleming AG, White BA, Haldorson GJ, Stone JB, Lessmann JJ, Eucker TP, Konkel ME (2015) The intestinal microbiota influences Campylobacter jejuni colonization and extraintestinal dissemination in mice. Appl Environ Microbiol 81:4642-4650

Ohsugi T, Kiuchi Y, Shimoda K, Oguri S, Maejima K (1996) Translocation of bacteria from the gastrointestinal tract in immunodeficient mice. Lab Anim 30:46-50
Peirano G, Ahmed-Bentley J, Woodford N, Pitout JD (2011) New Delhi metallo- $\beta$-lactamase from traveler returning to Canada. Emerg Infect Dis 17:242-244

Ratajczak M, Laroche E, Berthe T, Clermont O, Pawlak B, Denamur E, Petit $F$ (2010) Influence of hydrological conditions on the Escherichia coli population structure in the water of a creek on a rural watershed. BMC Microbiol 10:222

Rausch P, Basic M, Batra A, Bischoff SC, Blaut M, Clavel T, Gläner J, Gopalakrishnan S, GrassI GA, Günther C, Haller D, Hirose M, Ibrahim S, Loh G, Mattner J, Nagel S, Pabst O, Schmidt F, Siegmund B, Strowig T, Volynets V , Wirtz S, Zeissig S, Zeissig Y, Bleich A, Baines JF (2016) Analysis of factors contributing to variation in the C57BL/6 J fecal microbiota across German animal facilities. Int J Med Microbiol. doi:10.1016/j.jimm.2016.03.004

Sánchez S, Llorente MT, Echeita MA, Herrera-León S (2015) Development of three multiplex PCR assays targeting the 21 most clinically relevant serogroups associated with Shiga toxin-producing E. coli infection in humans. PLOS One 10:e0117660

Schmidt VM, Pinchbeck GL, Nuttall T, McEwan N, Dawson S, Williams NJ (2015) Antimicrobial resistance risk factors and charaterisation of faecal E. coli isolated from healthy Labrador retrievers in the United Kingdom. Prev Vet Med 119:31-40

Sengupta P (2013) The laboratory rat: relating its age with human's. Int J Prev Med 4:624-630

Skurnik D, Clermont O, Guillard T, Launay A, Danilchanka O, Pons S, Diancourt L, Lebreton F, Kadlec K, Roux D, Jiang D, Dion S, Aschard H, Denamur M, Cywes-Bentley C, Schwarz S, Tenaillon O, Andremont A, Picard B, Mekalanos J, Brisse S, Denamur E (2016) Emergence of antimicrobialresistant Escherichia coli of animal origin spreading in humans. Mol Biol Evol 33:898-914

Smati M, Clermont O, Bleibtreu A, Fourreau F, David A, Daubié AS, Hignard C, Loison O, Picard B, Denamur E (2015) Quantitative analysis of commensal Escherichia coli populations reveals host-specific enterotypes at the intraspecies level. Microbiol Open 4:604-615

Tenaillon O, Skurnik D, Picard B, Denamur E (2010) The population genetics of commensal Escherichia coli. Nat Rev Microbiol 8:201-217

Woodford N, Fagan EJ, Ellington MJ (2006) Multiplex PCR for rapid detection of genes encoding CTX-M extended-spectrum $\beta$-lactamases. J Antimicrob Chemother 57:154-155

Wu WJ, Sha SH, McLaren JD, Kawamoto K, Raphael Y, Schacht J (2001) Aminoglycoside ototoxicity in adult CBA, C57BL and BALB mice and the Sprague-Dawley rat. Hear Res 158:165-178

Wu H, Santoni-Ruqiu E, Ralfkiaer E, Porse BT, Moser C, Høiby N, Borregaard N, Cowland JB (2010) Lipocalin 2 is protective against E. coli pneumonia. Respir Res 11:96

\section{Submit your manuscript to a SpringerOpen ${ }^{\circ}$ journal and benefit from:}

- Convenient online submission

- Rigorous peer review

- Immediate publication on acceptance

- Open access: articles freely available online

- High visibility within the field

- Retaining the copyright to your article

Submit your next manuscript at $>$ springeropen.com 